\title{
How Biological Technology Should Inform the Causal Selection Debate
}

\author{
Janella Baxter*
}

\begin{abstract}
Waters's (2007) actual difference making and Weber's $(2013,2017)$ biological normality approaches to causal selection have received many criticisms, some of which miss their target. Disagreement about whether Waters's and Weber's views succeed in providing criteria that uniquely single out the gene as explanatorily significant in biology has led philosophers to overlook a prior problem. Before one can address whether Waters's and Weber's views successfully account for the explanatory significance of genes, one must ask whether either view satisfactorily meets the necessary conditions for causal selection in the first place. An adequate defense of causal selection must meet two desiderata. First, there must be an explanatory property that sets some causes apart from others. Second, the property identified must be one that is recognized by biologists as relevant to their domain(s) of inquiry. I argue that both fall short of meeting the second condition. I demonstrate this by showing how many of the biological technologies crucial to experimentation do not fit either view very well. I offer a more adequate proposal that accommodates non-actual and artificial causal variables. A consequence of my view is the following: When analyzing the causal selection practices of biologists, philosophers should consider the explanatory targets relevant to a research program-including ones whose explanans must appeal to biological technologies. I then explain how this proposal can inform the existing debate between Weber (2017) and Griffiths et al. (2015).
\end{abstract}

\section{Keywords}

causal selection $\bullet$ explanation $\bullet$ philosophy of biology $\bullet$ philosophy of technology $\bullet$ experiment

* Center for Philosophy of Science at the University of Pittsburgh, 1117 Cathedral of Learning, Pittsburgh, PA 15260, USA, jkb65@pitt.edu

Received 23 August 2018; Accepted 31 October 2018

doi:10.3998/ptpbio.16039257.0011.002 


\section{Introduction}

Causal selection is the widespread practice of singling out some causal variables from a set of causally relevant factors as explanatorily significant. The debate in the philosophy of biology literature has to do with what, if anything, justifies this practice in biology. Philosophers of biology have referred to the view that there is no explanatory justification for this practice as the causal parity thesis (Weber 2013). 1 Causal selection, by contrast, is the rejection of causal parity. On this view, biologists are at least sometimes justified in privileging some causes over others in explanation. Most contemporary philosophers of biology-even ones previously associated with causal parity - embrace this definition of causal selection (Griffiths et al. 2013; Griffiths et al. 2015; Woodward 2010; Waters 2007; Weber 2013; Stegmann 2012). Where disagreement abounds is the question of which causal variables biologists should systematically privilege in explanation and what serves as the underlying rationale. Two proposals that have attracted critical attention are C. Kenneth Waters's (2007) actual difference making account and Marcel Weber's $(2013,2017)$ biological normality account. However, some of the objections raised against these views have missed their target in one way or another.

Waters's actual difference making view states that causal variables whose actual varying in a population makes a difference to some trait are explanatorily significant. One criticism is that Waters treats "DNA sequences ... [as] the only causally specific actual difference maker with respect to the specificity of gene products" (Griffiths et al. 2013, 67). Critics who advance this objection have gone on to show that there are many other molecular variables that also serve as specific actual difference makers for gene products. The problem with this objection is that it mistakes Waters's paradigmatic example of an actual difference maker for a substantial view about all actual difference makers in biology. Yet Waters's actual difference making criterion is flexible and can apply to any type of variable that satisfies the requirements-not just genes. Hence, arguments that other biomolecules involved in determining the linear sequences of gene products need not be taken as a refutation of Waters's view. Instead, they succeed only in limiting the scope of Waters's paradigmatic example.

Another objection is that biologists appear to single out molecular genes for reasons other than actual difference making (Stegmann 2012). This objection appeals to the famous experiments carried out by Marshall W. Nirenberg and J. Heinrich Matthaei (1961) to decipher the genetic code. According to Ulrich Stegmann, some trials conducted by Nirenberg and Matthaei involved no actual genetic variation, yet the scientists singled out molecular genes as the explanatory variable. Stegmann takes this case as evidence that actual difference making doesn't adequately capture the explanatory significance of DNA and molecular genes in biology. Again, this objection mistakes Waters's paradigmatic example of an actual population-namely, a population raised and bred in the laboratory - as the only way to make sense of the actual difference making criteria. In fact, Waters's adopts a pluralistic view on what counts as an actual population that allows him to avoid this objection. He writes "biologists typically care only about whether a relationship holds under conditions actualized in organisms and their environments (or in the laboratory)" (Waters 2007, 576, italics mine.). Since "actual population" can refer to either a lab-controlled or naturally evolved population-populations outside experimental confines of a laboratory-genes can be actual difference makers in either. When Nirenberg and Matthaei privilege molecular genes in an experimental context, the relevant actual difference maker needn't be relative to the experimental population. It could instead be relative to a nat-

1. Some authors who embrace the label "causal parity" have systematically rejected this characterization (see Griffiths et al. 2013, chapter 4). This definition is nevertheless a useful conceptual tool for carving out the possible logical positions one can take.

๑ OPEN ACCESS - PTPBIO.ORG 
urally evolved population which the experimental population represents. Because genes often vary in actual populations outside of the lab, the Nirenberg and Matthaei case doesn't serve as a genuine counterexample.

Weber, by contrast, does defend the view that protein-coding genes are the primary explanatory variable in biology. The basis for his view is that molecular genes have the greatest amount of (relevant) fine-grained influence on the amino acid sequences of proteins. Critics of his view have attempted to identify other biomolecules that also determine the amino acid sequences of proteins - most notably, patterns of alternative splicing agents-but with even greater amounts of fine-grained influence than the relevant genes (Griffiths et al. 2015). The amount of fine-grained influence a causal variable has depends, importantly, on what types of fine-grained influence are recognized by biologists as relevant. Determining which causal variable has greater amounts of fine-grained influence requires that the same account of relevance is applied consistently to all candidate variables. Griffiths et al. (2015) consider only actual types of fine-grained influence as relevant, while Weber considers biologically normal types relevant. When these analyses are applied consistently, different causal variables end up having different estimates of fine-grained influence. Resolution of the debate can't be settled just by generating a quantitative estimate of a causal variable's amount of fine-grained influence. It requires that philosophers first settle which type of fine-grained influence is most relevant.

Importantly, disagreement about whether genes are the most explanatory variable in biology has led philosophers to overlook a prior problem. Before one can address whether Waters's and Weber's views successfully provide criteria that uniquely singles out genes as explanatorily significant, one must ask whether either view satisfactorily meets the necessary conditions for causal selection in the first place. This is the task of the present paper. I argue that both authors fall short of meeting all the requirements of an adequate account for causal selection in the biological sciences. For causal selection to be justified, (1) there must be an explanatory property that sets some causes apart from others and, (2) the property identified must be one that is recognized by biologists as illuminating relative to their domain(s) of inquiry. What biologists recognize as explanatory is often implicit to the theories and hypotheses they articulate as well as their experimental practices. Although Waters and Weber wish to take theoretical commitments and experimental practices seriously into their accounts of causal selection (Waters 1994, 2004, 2006; Weber 2004, 2018), I will argue that both authors fall short of meeting the second condition especially when it comes to the experimental practices of many researchers working in areas of molecular biology.

I construct my argument by showing that non-actual and artificial types of fine-grained influence are ubiquitous to the experimental and explanatory practices of many biologists. This is especially evident when biologists make and use technologies. The orthogonal tRNA technology developed by the Peter Schultz Lab (Wang et al. 2000) is a central case in my discussion because it serves as a direct counterexample to a phenomena Waters and Weber both claim to lie beyond the scope of biological inquiry. However, this is not the only technology I discuss. The same lessons I derive from the orthogonal tRNA case can be derived from green fluorescent protein and model organisms. By considering how biological technologies are developed and used in areas of contemporary molecular biology, I demonstrate the kind of fine-grained influence that biologists recognize as illuminating extends significantly beyond the actual and the biologically normal. In arguing my case, I also entertain and reject several objections concerning whether technologies like green fluorescent protein and orthogonal tRNA are, in fact, explanatory and relevant to molecular biologists. I show that even technologies developed by synthetic biologists satisfy the same criteria for explanatory significance as the actual and biologically normal variables that Waters and Weber recognize. The only difference is that the technologies I consider 
were at one point in scientific history merely potential and were made actual by artificial means. I argue that for philosophers who seek to inform their philosophies by taking experimental practices into account, the cases of biological technology I discuss serve as genuine counterexamples to the actual difference making and biological normality approaches.

Philosophers of biology who aim to analyze the causal selection practices of biologists should take seriously the role biological technology plays in explanation and experimentation. Thus, I propose an alternative view to better facilitate this. My view is that causes with fine-grained influence on life processes-whether this is achieved naturally or artificially-are experimentally and explanatorily significant in biology. When analyzing the causal selection practices of biologists, philosophers should consider the relevant explanatory targets of a particular research program-including ones that are brought about by biological technologies. The method I recommend has important consequences for the ongoing debate between Weber (2017) and Griffiths et al. (2015). Thus far, both sides have overlooked how relevant biological technologies bear on the quantitative estimates they have generated for measuring amounts of fine-grained influence. The biologists studying the phenomena appealed to by Weber and Griffiths et al. rely importantly on artificial types of fine-grained influence. Philosophers involved in this debate should reconceptualize the type of fine-grained influence that informs their quantitative estimates. It may turn out that the quantitative estimates they've reached should be revised. By not attending to the role biological technologies play in experimentation and explanation, philosophers risk mischaracterizing the nature of causal selection in biology.

I begin (Section 2) by laying out two desiderata that an adequate defense of causal selection must meet. Next, I describe (Section 3) the criteria for fine-grained influence. I continue (Section 4) by discussing a case from the history of biology in which biologists show a preference for some types of fine-grained influence. This case serves as the basis for Waters's (2007) and Weber's (2013) respective views on what falls within the scope of biology. Then I demonstrate (Section 5) how their views mischaracterize the sort of fine-grained influence that many biologists explanatorily privilege by discussing several counterexamples from biotechnology. Here I offer an alternative view, defend it against several objections, and develop some consequences of my argument.

\section{Causal Selection}

It's widely received that many causes are involved in any given effect. But biologists (and scientists more generally) don't cite each and every cause when formulating their hypotheses and theories. Instead biologists privilege some causes over others with respect to an effect of interest. This is made especially evident in many explanations where a variable (or set of variables) like a gene are singled out from all other relevant causal conditions as explanatorily significant with respect to, say, a phenotypic trait like eye color. Yet, this practice alone doesn't settle whether biologists are ever, in fact, justified in explanatorily privileging some causes over others. For the practice to be justified, two necessary conditions must be met.

Causal selection is a popular view in the philosophy of biology literature (Waters 2007; Weber 2013; Griffiths and Stotz 2013; Griffiths et al. 2015) according to which explanatorily privileging some causes over others isn't arbitrary but is (at least sometimes) justified on the basis of an explanatory distinction among causal conditions. Explanatory significance is analyzed in terms of explanatory properties that some factors possess with respect to an effect and that others lack. In the absence of any such property, causal selection is arbitrary and not principled. For one is equally justified in privileging some causal variable as any other. This is the first desideratum that an adequate defense of causal selection must meet - it must identify an explanatory property

๑ OPEN ACCESS - PTPBIO.ORG 
that sets some causes apart from others. There may be great variety in what the property is that distinguishes among causes, but nevertheless, there must be some such property for causal selection to be principled.

Yet if a plausible version of causal selection is to be defended there is a further desideratum that must be met and that has not been explicitly articulated. There are many explanatory properties that can distinguish among causal conditions, but not all of them are recognized by biologists as being relevant or illuminating of their domain(s) of inquiry. The search for an explanatorily distinguishing feature is motivated by an interest in capturing the explanatory practices that are unique to biology. The task for proponents of causal selection, then, isn't just to identify explanatory properties that set some causal factors apart from others, but also to identify properties that biologists recognize as genuinely illuminating and particularly relevant to their domain(s) of inquiry. This might initially appear like an appeal to capricious interests and values, but it needn't be. What biologists recognize as illuminating and relevant can (at least sometimes) track objective empirical features that characterize the reality they seek to understand. As we'll see, properly capturing the reality that many biologists seek to understand is the heart of this discussion.

Thus, an adequate defense of causal selection in biology must meet two desiderata. First, it must identify an explanatory property that sets some causes apart from others. This makes causal selection principled and not arbitrary. Second, biologists must recognize the property identified as illuminating and relevant to their domain(s) of inquiry.

\section{Fine-Grained Influence}

A variable with fine-grained influence is explanatorily distinguished from "switch-like" causes. While many types of causes can have the property of fine-grained influence, I will focus on a case that has received much attention in the causal selection literature-namely, the fine-grained influence some genes have on the amino acid sequences of proteins.

Woodward's (2010) criteria for fine-grained influence have been widely adopted as an analysis of causal selection in the philosophy of biology (Waters 2007; Griffiths et al. 2013; Griffiths et al. 2015; Weber 2013, 2017). Causal variables with fine-grained influence satisfy the first condition an adequate defense of causal selection must meet insofar as they possess an explanatory property that distinguishes them from "switch-like" causes. "Switch-like" causes can take one of two possible values- “on" or "off." By contrast, fine-grained causes can take any value from a range of possible alternative values. Consider the dial of a refrigerator thermostat whose six settings systematically correspond to one and only one temperature output. Setting the dial to 2 activates the cooling mechanism to bring the refrigerator to 2.5 degrees centigrade, while setting the dial to 3 raises the temperature to 5 degrees, and so on for each setting. The interventionist's rationale for privileging fine-grained causes over "switch-like" ones is that the former offer numerous opportunities to manipulate and control the value of an effect variable; whereas, the latter offers only two (Woodward 2010). Fine-grained causes can provide answers to a greater number of counterfactual questions than "switch-like" causal variables can.

Ideally, relationships with fine-grained influence will exhibit a perfect one-to-one mapping between input and output states, and every output will correspond to some input. ${ }^{2}$ However, the criteria for fine-grained influence may be weakened in various ways that are important for

2. In this way, fine-grained influence is like a bijective function. Bijective functions have one-to-one mappings between input and outputs states (injective) and every output state has some input (surjective). Also inherent to the criteria for fine-grained influence is the requirement that the level of detail used to describe a causal variable meet proportionality constraints as detailed in Woodward (2010). Proportionality ensures a partitioning of a causal 
modeling real world cases of fine-grained influence. Instead of requiring perfect one-to-one mapping between input and output states, some (but not too many) cases where more than one input state corresponds to the same output are permitted. Another way to weaken the criteria for fine-grained influence is to allow some (but again, not too many) output states to have no corresponding input. The idea that fine-grained influence is supposed to capture is of a causal variable that exerts a great degree of control over the states of an effect variable. Thus, it is not tolerable to the achievement of such control if the same input state maps onto more than one output or if many output states don't have some corresponding input. It is tolerable, however, to have more than one input associate with the same output. In the event that the same input state associates with more than one output, then the causal variable does not specify the effect state in a determinate way. And were there a number of effect states without a corresponding causal state, then our causal variable would have only a small degree of control over the system. Nevertheless, admitting a weakened criterion for fine-grained influence preserves the same general ideathese relationships display a range of alternative states where (for the most part) each causal state systematically associates with one effect state.

The criteria for fine-grained influence goes some way towards satisfying the second condition needed for an adequate defense of causal selection. It is an explanatory property many variables possess that biologists often privilege in explanation. Although protein-coding genes are not the only type of cause that has fine-grained influence on protein sequences-patterns of alternative splicing agents can also have this property (see Griffiths et al. 2013 and Griffiths et al. 2015) - they are a common example in the causal selection literature. A protein-coding gene consists of regulatory modules as well as a sequence of four nucleotide bases-adenine (A), thymine $(\mathrm{T})$, cytosine $(\mathrm{C})$, and guanine $(\mathrm{G})$ - along a segment of DNA. The same gene can have multiple variants or nucleotide sequences insofar as the same stretch of DNA is characterized by a different sequence of As, Ts, Cs, and Gs in different populations. Not all possible gene variants actually obtain in evolved populations. Nevertheless, in principle, every gene can have a large number of different nucleotide sequences such that each sequence is analogous to a different setting on a thermostat dial.

For the most part, each nucleotide sequence systematically associates with one and only one protein just as each setting on the thermostat systematically corresponds to a single temperature output. A gene determines the sequence of amino acids in units of three nucleotides or codons. All 20 canonical amino acids are specified by a codon. ${ }^{3}$ But there is some redundancy in the genetic code because more than one codon can specify the same amino acid-for instance, UUU and UUC both specify the amino acid phenylalanine. ${ }^{-1}$ It is true of other amino acids that more than one codon specifies them. This means that there will be some gene variants that determine the same amino acid sequence of a protein. But this is permitted on the weakened criteria of fine-grained influence insofar as such relationships need not be perfectly one-to-one.

Philosophers of biology widely regard fine-grained influence as an adequate analysis of causal selection. But are all possible instantiations of fine-grained influence recognized as significant by biologists? This question turns out to be harder to answer than one would expect, because biologists have historically privileged some types of fine-grained influence over others.

variable into alternative states that do not overlap and that pair with one and only one alternative state of an effect variable.

3. There are 22 amino acids that form proteins in all. Selenocysteine and pyrrolysine require special incorporation mechanisms that involve structural motifs of the mRNA sequence in addition to a codon.

4. Coding and informational metaphors abound in biology, the meaning and significance of which varies in different contexts. A number of philosophical analyses of this language exist. (Godfrey-Smith 2000; Griffiths et al. 2015) For my purposes, what I mean by "genetic code" is only the minimal association of codon to transfer RNA to amino acid. 
Although there is consensus among philosophers that the criteria for fine-grained influence meet the desiderata for causal selection, there is disagreement about how exactly they satisfy the second requirement.

\section{Privileged Fine-Grained Influence}

Historically, biologists have ruled out some types of fine-grained influence as interesting because they did not think such cases were representative of life in our world. In particular, many biologists have not regarded the potential fine-grained influence of transfer RNA (tRNA) as illuminating the kind of processes and systems they study (Osawa et al. 1992; McCutcheon et al. 2009). As I will show, this attitude is a consequence of Francis Crick's (1968) frozen accident theory about the genetic code. This example from the history of biology shows that satisfaction of the criteria for fine-grained influence isn't sufficient for biologists to recognize a variable as explanatorily significant. The criteria must be supplemented with an account of the type of finegrained influence that biologists regard as relevant and explanatory. In an effort to provide such an account, Waters (2007) and Weber (2013) have proposed that biologists privilege actual and natural cases of fine-grained influence respectively.

Although biologists have acknowledged for some time that tRNA molecules have potential fine-grained influence on protein sequences, it has been common to dismiss this feature as irrelevant to the study of biology. This is because biologists have long regarded tRNA as a mediator of the mechanism by which genes have fine-grained influence in populations and not as something that has any fine-grained influence of its own. This common scientific attitude has influenced Waters's (2007) and Weber's (2013) respective views on what falls within the scope of biology. But before we can understand the attitudes regarding the status of tRNA, we need a general understanding of how tRNA has potential fine-grained influence.

The activity of tRNA enters at the stage of protein synthesis called translation. The nucleotide sequences of protein-coding genes are copied into mRNA, whose "information" is then translated into a sequence of amino acids. Translation is carried out by an enzyme complex called the ribosome, which reads the nucleotide sequences of the mRNA and binds amino acids together in the instructed order. The tRNA molecules deliver amino acids to the ribosome in the order specified by the mRNA. To translate the genetic code with fidelity, tRNA works together with tRNA synthetases to match the correct amino acid to the correct codon of the mRNA. With the exception of "stop codons"-codons that instruct the process of translation to stopthere is a type of tRNA/tRNA synthetase pair for every type of codon (amounting to about 61). Each type of tRNA carries one and only one type of amino acid. For instance, there is a tRNA that only recognizes the UUU codon in mRNA and only delivers phenylalanine. Thus, there are sequences of nucleotides (or codons) in an mRNA that correspond to sequences exhibited by tRNAs and, in accordance with the instructions of the mRNA, help synthesize a sequence of amino acids to eventually form a protein.

Biologists have known that there is variation in a tRNA's amino acid assignment, but such cases were regarded as exceptions relegated to very specific environments. Mitochondria and chloroplasts were once single-celled species that have evolved to be organelles whose operations are now integral to the proper functioning of eukaryotic cells. They have since lost much, though not all, of their genomes. Although the relationship between these membrane-bound organelles and eukaryotic cells is symbiotic, these organelles have some of their own functions independent of the rest of the cell. For example, one function is the encoding and synthesizing of their own proteins. What's exceptional about some species of mitochondria and chloroplasts (and some types of viruses) is that they possess a nonstandard genetic code-or a nonstandard codon- 
tRNA-amino acid assignment. In these cases, what normally functions as a "stop codon" (e.g., UGA) in the standard genetic code, is assigned an amino acid (Barrell et al. 1979). This is an example of variation in the tRNA's amino acid preference. However, biologists have long believed that the majority of genomes obey the standard genetic code, according to which the UGA codon instructs protein synthesis to stop.

The genetic code, which relates DNA sequence to protein sequence, is nearly universal across all life. Examples of recodings do exist, but new instances are rare. Genomes that exhibit recodings typically have other extreme properties, including reduced size, reduced gene sets, and low guanine plus cytosine (GC) content. The most common recoding event ... was previously known for several mitochondrial and one bacterial lineage ....” (McCutcheon et al. 2009)

Thus, biologists regarded the nonstandard genetic code of some organisms to be rare cases that don't accurately represent the majority of living systems.

Many biologists have assumed that tRNA does have fine-grained influence but only with respect to a range of interventions and background conditions that aren't actual or manifested in living systems. In these other contexts, the amino acid assignment of each tRNA may be altered-tRNA that normally carries proline now carries glycine, tRNA that normally carries glycine now carries proline, and so on for all 61 types of tRNA. In this scenario, the same genetic sequence can now associate with different sequences of tRNA (depending on the amino acid assignment) - a range of sequences $\left(\beta_{1}, \ldots, \beta_{n}\right)$ for each possible alternative tRNA-amino acid assignment. Just as each possible nucleotide sequence of a gene is analogous to an alternative "setting" on the thermostat dial, so is each $\beta_{i}$. But, importantly, many biologists believe that there is no variation (at least in most genomes) in the actual codon-tRNA-amino acid pairings of the majority of evolved populations in our world. From bacterium to elephant, each type of tRNA associates with its assigned amino acid and nothing else. Instead, a gene with a nucleic acid sequence specifies one and only one sequence of tRNAs—say, $\beta$ - which in turn maps onto one and only one protein sequence.

The belief that there is no variation in the actual codon-tRNA-amino acid pairings of life in our world is a consequence of Francis Crick's (1968) widely accepted "frozen accident" theory. The "frozen accident" theory is the idea that the systematic assignment between codon-tRNAamino acid is a contingent and life-sustaining fact about our world. Each codon's association with a particular tRNA and amino acid could have been otherwise. But, as a matter of fact, the genetic code is frozen-or unchanging-across the populations and evolutionary histories of our actual world (Osawa et al. 1992). Crick once wrote:

This theory states that the code is universal because at the present time any change would be lethal, or at least very strongly selected against. This is because all organisms (with the possible exception of certain viruses) the code determines (by reading the mRNA) the amino acid sequences of so many highly evolved protein molecules that any change to these would be highly disadvantageous unless accompanied by many simultaneous mutations to correct the 'mistakes' produced by altering the code." (Crick 1968, 370)

According to this hypothesis, variation to the standard genetic code would be lethal to the living system. This is because numerous life-sustaining metabolic processes-such as protein synthesis, cellular differentiation and respiration, apoptosis, etc.- - operate according to the rules of the genetic code that have evolved in our world. Any deviation from these instructions will interfere 
with the complex processes that sustain life. Alternative genetic codes and tRNA-amino acid assignments, while possible, could only be viable in systems that have evolutionary histories vastly different from our own. Thus, the general attitude has been that nonstandard codes (A) do not characterize the actual living systems of our world and (B) artificial nonstandard genetic codes would likely be lethal to an experimental population, thereby making them unilluminating.

So far, we've seen that satisfying the criteria for fine-grained influence alone isn't sufficient for researchers to recognize a causal variable as relevant and explanatory. This suggests that the criteria must be supplemented with an account of the type of fine-grained influence researchers do recognize as relevant and illuminating. Waters and Weber both point to the potential finegrained influence of tRNA as the sort of fine-grained influence that biologists don't prefer. Waters, for instance, writes that varying the codon specificity of tRNA "does not matter to biologists unless different tRNA [have] actually existed or is likely to exist actually in the future" (Waters 2007, 576). Similarly, Marcel Weber asserts in reference to the potential fine-grained influence of tRNA that biologists "don't care about what might happen in some highly contrived possible worlds" (Weber 2013, 33).

Although Waters and Weber rely on the same example, they offer different characterizations of the sort of fine-grained influence they think biologists do prefer. Waters draws from experimental practices to analyze the nature of explanation and investigation in classical and molecular genetics. He asserts in several places that biologists are principally concerned with studying and explaining causal relationships that actually, as opposed to potentially, obtain. 5 This, he claims, is the distinguishing feature between biology and the physical sciences:

$[\mathrm{w}]$ hereas physical scientists often try to establish generalizations that express causal relationships that hold over conditions that have not and will not be actualized (to their knowledge), biologists typically care only about whether a relationship holds under conditions actualized in organisms and their environments (or in the laboratory)." (Waters 2007, 576)

Weber also draws from the experimental practices of biologists in an effort to construct philosophical analyses of causal explanation (Weber 2004, 2018). Unlike Waters, Weber recognizes that biologists do regard some types of potential difference making causes as explanatorily significant. Instead, he appeals to the "frozen accident" theory to rule out some possibilities as too "mad or gerrymandered" to be illuminating of the phenomena biologists wish to explain (Weber 2017). He notes that even though variation in the amino acid assignment of tRNA is possible, it nevertheless lies beyond the scope of biological inquiry. For such a possibility to be made actual in a way that is not lethal to the living system, it would have to be achieved by artificial and "surgical" means. Such means, he asserts, would surely be unilluminating. Thus, he insists that the type of fine-grained influence that matters to biologists are types that "(1) .. may also be due to natural processes ... and (2) [are] compatible with the continued persistence of the biological entity that is being considered" (Weber 2013, 27).

5. Weber (2013) and Currie (2018) also criticize Waters for under-appreciating the explanatory and investigative significance of potential difference makers. Currie draws from the historical sciences to effectively make his claim. Yet, Waters's principal aim is to characterize the explanatory and investigative practices of scientists working in areas of genetics and molecular biology. Thus, a proponent of the actual difference making account can grant Currie's claim, and nevertheless maintain that the actual/potential distinction is appropriate for the areas of biology Waters wishes to address. 
For both authors, the paradigm example of a phenomena that lies beyond the scope of biological inquiry are non-standard genetic codes achieved by varying the amino acid preference of tRNA. For Waters, variation in the amino acid assignment of tRNA does not actually occur to any significant degree in most living things-outside of the exceptional cases (like some membrane-bound organelles) mentioned previously. While for Weber, it does not satisfy the conditions for biological normality.

According to Waters and Weber, biologists prefer either whatever actually obtains or whatever satisfies biological normality. They reach these views by treating the potential fine-grained influence of tRNA as a paradigm example of a phenomenon that lies beyond the scope of biological inquiry. Echoing a common attitude within biology, Waters and Weber maintain that the potential fine-grained influence of tRNA is not of interest to biologists; it does not possess the kind of fine-grained influence that researchers working in areas of molecular biology privilege.

\section{Biological Technology in Explanation and Practice}

Whether one adopts Waters's or Weber's characterization of the sort of fine-grained influence that is privileged in biology, there are problems with their views. Both have trouble accounting for new technological advancements in biology. Making new things happen by means of artificial techniques is a practice indispensable to experimental approaches in molecular biology and genetics. I argue in this section that biological technologies are explanatorily significant for the artificial effects they produce. I entertain several objections that challenge whether technology is explanatorily relevant for actual, naturally evolved phenomena. In addressing this challenge, I show how biological technologies are often indispensable for the inferences made about the actual, naturally evolved processes under scrutiny. For practice-oriented philosophers like Waters and Weber, the indispensable role that biological technologies play in investigation and experimentation should be accommodated by an adequate account of causal selection. The counterexamples discussed in this section suggest that Waters and Weber have mischaracterized the kind of fine-grained influence that many biologists regard as relevant and illuminating. Here I offer an alternative view according to which biologists privilege fine-grained influence- bowever this is achieved - as explanatorily and investigatively significant. I then show how my proposal can inform the existing debate between Weber (2017) and Griffiths et al. (2015).

While biologists make use of a variety of biological technologies, the artificial variation in the codon specificity of tRNA is especially relevant for the current discussion. A number of research programs have attempted varying actual and natural codon-tRNA-amino acid assignments as a way to incorporate unnatural amino acids ${ }^{6}$ into proteins. However, many of these programs have faced serious limitations. One strategy (solid-phase peptide synthesis) produces a chain of amino acids in a series of steps whereby the reactive groups of each amino acid are blocked/unblocked at precise moments during the process. This ensures that the C-terminal of an amino acid binds only to the N-terminal of another (Kimmerlin et al. 2005). Although this method gives researchers precise control over the amino acid sequence of a protein, it is challenging to synthesize proteins that are made in living systems. On average, naturally produced proteins are longer than the proteins made by solid-phase synthesis, which are limited to 50-100 amino acids in length. Another problem is that this process cannot be carried out in living cells, making it difficult to study protein structure and function of living systems. Another approach employs living cells that are auxotrophic for a canonical amino acid-that is, the pathway necessary for synthesizing the amino acid is turned "off" in the cell. The cell's natural

6. Unnatural amino acids, such as $\mathrm{p}$-Acetylphenylalanine or $\mathrm{p}$-Fluorophenylalanine, are canonical amino acids whose side-chain ( $\mathrm{R}$ group) has been modified in novel ways.

๑ OPEN ACCESS - PTPBIO.ORG 
tRNAs are then forced to incorporate an unnatural amino acid to replace the type the cell does not make (Budisa et al. 1999). This method successfully incorporates unnatural amino acids into the protein synthesis processes of living systems; however, it also has limitations. The incorporation of unnatural amino acids is not site-specific but is a global replacement of an amino acid in all proteins. This can be toxic to the cell. Furthermore, the method is limited to using unstable biological systems - systems that cannot sustain cellular growth and division under normal conditions.

However, a much more efficient and precise method for the site-specific incorporation of unnatural amino acids into the protein synthesis processes of living cells was eventually accomplished by synthetic biologists at the Peter Schultz Lab (Wang et al. 2000). Schultz and associates successfully varied the codon specificity of tRNA by engineering orthogonal tRNA. Orthogonal tRNA molecules are exogenous to the biological system of interest and are artificially modified to systematically associate a specific type of unnatural amino acid with a "stop codon." This technology achieves a level of control and precision lacked by previous methods. Like the mitochondria and chloroplasts discussed above, orthogonal tRNA successfully varies the codon-tRNA-amino acid assignment; however, orthogonal tRNAs carry out their function in living organisms (like bacteria, yeast, and mammalian cells) that would otherwise obey the instructions of the standard genetic code (Liu et al. 2010). By inserting a stop codon into any region of a gene, researchers can now insert an unnatural amino acid into (almost) any part of a protein. A wide array of unnatural amino acids can now be assigned to a tRNA that recognizes "stop codons," thereby creating an arbitrary number of alternative tRNA sequences for any given mRNA sequence. This technology has effectively made the potential fine-grained influence of tRNA actual.

Unnatural biological variants are pervasive in much of biology research. Consider the gene for green fluorescent protein (GFP) and model organisms. Genes for green fluorescent protein originated in various marine organisms and are often used as a tool for tracing gene expression in a variety of non-marine organisms (like mice, chicks, round worm, etc.). When used as an observational tool, the gene for GFP is fused to the target gene such that a fused protein-a protein expressing the genes for both the target protein and GFP-is expressed. The expression of a GFP-fused protein helps researchers infer when (and how much of) the target gene is being expressed. This technology has been so effective that a Nobel Prize was awarded in 2008 for its development (Chalfie et al. 1994). Importantly, the GFP variant biologists use differs from the variants found in nature. The gene sequence has been modified to optimize and diversify its spectral properties (Heim et al. 1995; Stepanenko et al. 2008). While variation in a gene's sequence is both natural and actual, the GFP many researchers use in the lab may not be obtainable by natural processes in the non-marine organisms in which it is used (e.g., by random mutation). And, even if it were to arise naturally, the expression of the gene might lower the population's overall fitness so as to prevent the trait from accumulating in later generations. Similarly, model organisms - whose use is commonplace across biology labs-differ in important ways from the natural populations they are supposed to represent (Ankeny et al. 2011). Model organisms, like the fruit fly (Drosophila), the bacterium E. coli, and baker's yeast (Saccharomyces cerevisiae), serve as representative specimens for a broader class of species like human beings, such that insights

7. Specifically, tRNA work together with aminoacyl-tRNA synthetases to identify the correct amino acid. The Schultz lab also had to engineer orthogonal aminoacyl-tRNA synthetases to carry out the same function for the orthogonal tRNA.

8. Making a technological possibility a reality is no trivial endeavor. There is no guarantee that the technological possibilities researchers imagine can be made a reality even with the best engineering efforts available at a point in history. Over the course of the Schultz Lab's effort to make orthogonal tRNA the researchers encountered unexpected hurdles that they did not know a priori could be overcome. 
about the former may enhance our understanding about the latter. Model organisms undergo many rounds of controlled breeding regimens to produce relatively genetically uniform populations. This uniformity is often crucial for studying important metabolic functions and processes; however, this feature is unlikely to be adaptive in environments outside of the lab.

One might object that biological technologies are merely tools for developing knowledge about the biological processes they make observable rather than explanations of such processes. GFP, for instance, doesn't explain the activity of the target gene. Instead, it is the biological processes involved in gene expression that explains the presence/absence of the target gene. GFP merely makes the process visible.

But observational tools like fluorescent genes are often invoked in biological explanations for having fine-grained influence on the artificial effects they produce. The nucleic acid sequence of a fluorescent gene has some amount of fine-grained influence on the spectral properties that are crucial for inferring things about the biological process that is the target of a study (Stepanenko et al. 2008). Alternative nucleic acid sequences encoding multiple nonsynonymous amino acid substitutions (some including three or more) systematically associate with different fluorescent colors. 9 When researchers explain what makes the difference between, say, cyan and yellow fluorescence in a given experiment, they single out the different nucleic acid sequences that encode the fluorescent proteins. 10 The fine-grained causal relationship between the gene and the artificial effects it produces-in this case, the color of fluorescence-is often crucial for inferences researchers make about the target processes they wish to investigate. The target process(es) a biologist wishes to study do not always produce easily observable effects. So, the process by which an easily observable correlate is achieved matters greatly for the biologist's reasoning about the target process. When a researcher observes one type of fluorescence rather than another in an experimental system, they may reliably infer that a gene of interest along with the relevant fluorescent gene have been expressed. Artificial causes are explanatorily significant for the effects they produce. Reliance on the causal relationship between technology and artificial effect is an indispensable method in experimental situations where a researcher would not otherwise have knowledge about the target process they seek to study.

A related concern might be that there is an important asymmetry between the kind of technology that orthogonal tRNAs are and the kind of technology more traditional observational tools are. GFP and model organisms may be explanatory, but orthogonal tRNAs are not. After all, the former technologies were developed for the purposes of enhancing the study and explanation of actual living systems. However, orthogonal tRNA is a tool developed and used for advancing research programs in synthetic biology. Contemporary synthetic biology is distinctive from the rest of biology in that it does not aim to produce knowledge and understanding about actual, naturally evolved biological systems, but instead aims to engineer novel ones (Keller 2009; Endy 2008). In fact, a major use to which the orthogonal tRNA technology is put is to the production of synthetic organisms that synthesize pharmaceuticals, like antibody-drug conjugates (Axup et al. 2012). It may be that some technologies are explanatorily significant in the biological sciences, but the case of orthogonal tRNA is not of the relevant type.

9. The amount of fine-grained influence that alternative fluorescent protein-coding genes have with respect to fluorescent colors will be a proper subset of the amount of fine-grained influence the same gene will have on the amino acid sequence of a protein. Furthermore, it is likely more than one alternative nucleic acid sequence will associate with the same color output. This is permissible, however, on Woodward's formal criteria for fine-grained influence. An analogous point applies to the orthogonal tRNA case.

10. Gene expression patterns play a role in whether a fluorescent gene is expressed at all and the intensity of fluorescence that obtains. The point here is that the nucleic acid sequence of the gene must feature in explanations of the different fluorescent colors. 
Much of what has been said in the case of fluorescent genes can be said in defense of the orthogonal tRNA technology. When biologists wish to explain the presence of an unnatural amino acid in a novel protein produced by the Schultz technology, they must appeal to the relevant orthogonal tRNA. Initially one might think that the incorporation of an unnatural amino acid in a protein is explained by the corresponding "stop codon" that has been "rewritten." However, the stop codon doesn't satisfy the criteria for fine-grained influence. This is because the "stop codon" systematically associates with two outputs. Sometimes it serves as a standard "stop codon," other times it associates with the unnatural amino acid. This is not permitted on the criteria for fine-grained influence. Each alternative value in a causal variable must associate with one and only one value in an effect variable. When it comes to explaining the unnatural amino acids in proteins, the causal variable that satisfies fine-grained influence are the tRNA molecules. There are a range of alternative orthogonal tRNAs, each of which systematically associate with a unique unnatural amino acid. What explains the difference between one unnatural amino acid and another being present in a protein is the difference between alternative orthogonal tRNA molecules. Moreover, like fluorescent proteins, the orthogonal tRNA technology can aid in our study and explanation of naturally evolved phenomena. An important use to which the orthogonal tRNA technology is put is the study of naturally evolved protein structure and conformational change. A range of alternative orthogonal tRNA molecules can be used to sitespecifically incorporate a range of unnatural amino acids with distinctive resonance frequencies (Cellitti et al. 2008). Detection of the distinctive resonance frequencies produced by unnatural amino acids is often crucial for making inferences about the conformational change of an otherwise naturally evolved protein. The use of biological technologies for observational purposes is a crucial part of experimental practices and the means by which biologists reach explanations of naturally evolved phenomena.

For authors seeking to inform their philosophies by drawing from the role of technology in science (like Waters and Weber), an account of causal selection requires a more adequate analysis of the type of fine-grained influence that many biologists privilege. The actual difference making and biological normality accounts both mischaracterize this in different ways. Waters's actual/potential distinction cannot differentiate between things that are potential merely because of the state of technology at a point in time, and things that are potential because they cannot in principle be realized in a living system. It is often the case-as with orthogonal tRNA technology and green fluorescent protein-that there is a period of time during which a biological technology has not yet been actualized and is merely potential. Yet, substantial research efforts are often necessary for making the technology a reality. During this period of scientific investigation, biologists must imagine and hypothesize the non-actual to a significant extent. When it comes to scientific investigation, Waters is mistaken-biologists don't "typically care only about whether a relationship holds under conditions actualized in organisms and their environments (or in the laboratory)" (Waters 2007, 576). They also care greatly about what is biologically potential at a point in scientific history.

Weber's proposal also gets the full scope of relevant possibilities wrong. The biological normality view recognizes a range of possibilities that is broader than the range that is recognized by the actual difference making view. For biological normality includes non-actual possibilities that can obtain by natural means. Yet the full scope of possibilities relevant to biological inquiry is even broader than this. The biological technologies I've discussed represent possibilities that are realized by human efforts in laboratory contexts. One might be tempted to argue that 
non-standard codon-tRNA-(synthetic) amino acid assignments 11 could evolve by some natural process outside of the laboratory, in which case the orthogonal tRNA case would be biologically normal. But this sort of claim is not well-defined without any specification as to the number of mutations, evolutionary time scale, and environmental conditions needed to bring about and maintain a synthetic genetic code. ${ }^{12}$ It is unlikely that already existing organisms would evolve pathways that synthesize novel amino acids as well as the necessary tRNA/tRNA amino-acyl synthetases on an evolutionary time scale that is meaningful. Thus, when it comes to the making and using biological technologies, the range of possibilities biologists recognize as relevant and illuminating is often broader than what can be achieved by biologically normal means.

The central problem with Waters's and Weber's views is that the kind of fine-grained influence that many biologists recognize as relevant and illuminating is not restricted to what is actual and biologically normal. Thus, a more nuanced analysis is needed. As an alternative, I propose the view that biologists recognize causes with fine-grained influence on life processes-however this is achieved - as explanatorily significant. As I've discussed, the use and development of biological technologies reveals that many biologists regard causes with fine-grained influence as illuminating and relevant when the ability to study and manipulate life processes is enhancedregardless of whether fine-grained influence is achieved by natural or artificial means. For finegrained causal variables to enhance the study and manipulation of a life process, the variable and its effects often must be capable of integration with the life-sustaining processes of the living entity in question - at least to some degree. For example, novel proteins that are achievable only by artificial processes are unlikely to be relevant or illuminating to many biologists if they degrade immediately, are severely misfolded, have no function, or have a serious deleterious function. This is why protein engineering through solid-phase peptide synthesis and auxotrophic methods have not been satisfying to many researchers. Nevertheless, there are many engineered protein sequences that can integrate with the life processes of a living thing to some degree of success. Experimental versions of green fluorescent protein that are inserted into most model organisms and proteins synthesized with the aid of orthogonal tRNA are some examples. So, not all possible invariant relationships are of explanatory significance to biologists-as Weber correctly observes. But, the type of invariant relationships many biologists do regard as illuminating and relevant extends significantly beyond that of the biologically normal and the actual. However, my proposal isn't to identify the kind of fine-grained influence that is relevant to biology with Woodward's criterion for fine-grained influence. What types of fine-grained influence are relevant to biology is historically contingent and relative to particular research programs. Some types will be relevant to some research programs and not others. My recommendation is that philosophers attend carefully to the differences between research programs.

When philosophers seek to analyze the causal selection practices of biologists, they should take seriously the explanatory targets at play in a particular research program-including ones whose explanans must feature biological technologies. This can make a difference to which causal variables philosophers identify as genuinely explanatory. The current debate between Weber and Griffiths et al. (2015), for instance, hinges on how much fine-grained influence

11. Genome sequencing of wild microbial populations has recently found non-standard genetic codes that associate stop codons with naturally occurring amino acids to be much more common than had previously been thought (Ivanova et al. 2014).

12. Indeed, Weber attempts to meet this demand. He proposes to define biological normality in terms of the number of mutations needed to achieve two independent non-synonymous amino acid substitutions at different locations in a protein. His reasoning is that two non-synonymous amino acid substitutions commonly occur within just a few generations (Weber 2017). Note, however, that many artificial gene sequences that matter greatly to experimenters (a case in point are genes for fluorescent proteins) often contain more than just two non-synonymous amino acid substitutions. 
patterns of alternative splicing agents have on the unique Dscam protein in Drosophila. So far, the literature has only considered actual and biologically normal variants of alternative splicing (Weber 2017; Griffiths et al. 2015). Yet, the experimenters investigating this phenomenon employ artificial, chimeric variants as well (Wu et al. 2012). The artificial methods employed by these researchers help explain the artificial effects they achieve with chimeric Dscam proteins, which in turn are indispensable for the inferences they make about the naturally evolved system. Philosophers in this debate have not examined how this bears on the way they have conceptualized the type of fine-grained influence that is relevant to this case, nor have they considered how this should inform the quantitative estimates they produce. In overlooking the explanatory and investigative significance of biological technologies, philosophers are likely to mischaracterize scientific reasoning as well as the nature of the causal variables that are genuinely important.

\section{Conclusion}

Waters's actual difference making approach and Weber's biological normality approach to causal selection have received numerous criticisms, many of which fall short of presenting genuine challenges. This is because the philosophical literature has overlooked a prior question that any adequate account of causal selection must meet. Before one can ask whether Waters's and Weber's respective views succeed in providing a criterion that uniquely singles out genes in explanation, one must ask whether their views meet the necessary conditions for causal selection. Not only must an adequate account identify some explanatory property that sets some causes apart from others, but the property identified must also be one that biologists recognize as relevant and illuminating to their domains of inquiry. What biologists regard as relevant and illuminating is often implicit to their theoretical commitments and, importantly, their experimental practices. By not attending explicitly to this further requirement, Waters and Weber-who seek to inform their philosophies by attending carefully to the experimental practices of the sciences they study - do not adequately accommodate the role biological technologies play in causal selection.

I advocate that philosophers working on the causal selection debate should take seriously the explanatory targets that are relevant to a research program-including ones whose explanans must appeal to biological technologies. Not accommodating for these sorts of explanatory targets risks an incomplete understanding of how biologists working in genetics and areas of molecular biology reason. It also risks misidentifying the causal variables that are genuinely explanatory. The current debate between Waters (2013) and Griffiths et al. (2015) has not taken into account the artificial variants that are relevant to the labs studying alternative splicing of the Dscam protein in Drosophila. These authors have not considered how this might bear on the quantitative estimates they have reached, nor have they considered how this bears on the way they should conceptualize the type of fine-grained influence that matters to this research. The arguments of this paper might shed light on how this argument can move forward.

\section{Acknowledgments}

I would like to thank David Hilbert, Ken Waters, Marcel Weber, Alan Love, and several anonymous reviewers for their invaluable feedback on this article. Work on this project was supported by the Minnesota Center for Philosophy of Science and a grant from the John Templeton Foundation (ID 50191). This paper is dedicated to Elaine Ruth Baxter. 


\section{Literature cited}

Ankeny, R., and S. Leonelli. 2011. "What's So Special About Model Organisms?” Studies in History and Philosophy of Science 42: 313-323.

Axup, J., K. M. Bajjuir, M. Ritland, B. Hutchins, C. Kim, S. Kazane, R. Halder, et al. 2012. "Synthesis of Site-Specific Antibody-Drug Conjugates using Unnatural Amino Acids." Proceedings of the National Academy of Sciences 109 (40): 16101-6.

Barrell, B. G., A. T. Bankier, and J. Drouin. 1979. "A Different Genetic Code in Human Mitochondria." Nature 282: 189-194.

Budisa, N., C. Minks, S. Alefelder, W. Wenger, F. Dong, L. Moroder, and R. Huber. 1999. “Toward the Experimental Codon Reassignment in vivo: Protein Building with an Expanded Amino Acid Repertoire." Federation of American Societies for Experimental Biology 13 (1): 41-51.

Cellitti, S., D. H. Jones, L. Lagpacan, X. Hao, Q. Zhang, H. Hu, S. Brittain, et al. 2008. "In vivo Incorporation of Unnatural Amino Acids to Probe Structure, Dynamics, and Ligand Binding in a Large Protein by Nuclear Magnetic Resonance Spectroscopy." Journal of American Chemical Society 130: 9268-9281.

Chalfie, M., Y. Tu, G. Euskirchen, W. Ward, and D. Prasher. 1994. "Green Fluorescent Protein as a Marker for Gene Expression." Science 263: 802-5.

Crick, F. 1968. “The Origin of the Genetic Code.” Journal of Molecular Biology 38: 367-79.

Currie, A. 2018. Rock, Bone, and Ruin: An Optimist's Guide to the Historical Sciences. MIT Press.

Endy, D. 2008. "Engineering Biology: A Talk with Drew Endy." https://www.edge.org/conversation/drew_endy-engineering-biology

Godfrey-Smith, P. 2000. "On the Theoretical Role of 'Genetic Coding'.” Philosophy of Science 67: 2644.

Griffiths, P., and K. Stotz. 2013. Genetics and Philosophy: An Introduction. New York: Cambridge University Press.

Griffiths, P., A. Pocheville, B. Calcott, K. Stotz, H. Kim, and R. Knight. 2015. "Measuring Causal Specificity." Philosophy of Science 82 (4): 529-55.

Ivanova, N., P. Schwientek, H. Tripp, C. Rinke, A. Pati, M. Huntemann, A. Visel, T. Woyke, N. Kyrpides, and E. Rubin. 2014. "Stop Codon Reassignments in the Wild." Science 344 (6186): 909-13.

Keller, E. F. 2009. "Knowing as Making, Making as Knowing: The Many Lives of Synthetic Biology." Biological Theory 4 (4): 333-339.

Kimmerlin, T., and D. Seebach. 2005. "100 Years of Peptide Synthesis': Ligation Methods for Peptide and Protein Synthesis with Applications to $\beta$-peptide Assemblies." Journal of Peptide Research 65: 229-260.

Liu, C., and P. Schultz. 2010. "Adding New Chemistries to the Genetic Code." Annual Review of Biochemistry 79: 413-44.

McCutcheon, J., B. McDonald, and N. Moran. 2009. "Origin of an Alternative Genetic Code in the Extremely Small and GC-Rich Genome of a Bacterial Symbiont." Public Library of Science Genetics 5 (7): 1-11.

Nirenberg, M., and J. Matthaei. 1961. "The Dependence of Cell-Free Protein Synthesis in E. coli upon Naturally Occurring or Synthetic Polyribonucleotides." Proceedings of the National Academy of Sciences 47: 1588-1602. 
Osawa, S., T. Jukes, K. Watanabe, and A. Muto. 1992. "Recent Evidence for Evolution of the Genetic Code." Microbiological Reviews 56 (1): 229-264.

Stegmann, U. 2012. "Varieties of Parity." Biology \& Philosophy 27: 903-918.

Stepanenko, O., V. Verkhusha, I. Kuznetsova, V. Uversky, and K. Turoverov. 2008. "Fluorescent Proteins as Biomarkers and Biosensors: Throwing Color Lights on Molecular and Cellular Processes.” Current Protein Peptide Science 9(4): 338-369.

Wang, L., T. Magliery, D. Liu, and P. Schultz. 2000. "A New Functional Suppressor tRNA/Aminoacyl-tRNA Synthetase Pair for the in Vivo Incorporation of Unnatural Amino Acids into Proteins." The Journal of American Chemistry Society 122: 5010-11.

Waters, C. K. 1994. “Genes Made Molecular.” Philosophy of Science 61: 163-185.

Waters, C. K. 2004. "What Was Classical Genetics?" Studies in History and Philosophy of Science, 35: 783-809.

Waters, C. K. 2006. "How Practical Know-How Contextualizes Theoretical Knowledge: Exporting Causal Knowledge from Laboratory to Nature.” Philosophy of Science 75 (Proceedings): 707-719.

Waters, C. K. 2007. “Causes That Make a Difference.” Journal of Philosophy 104 (11): 551-579.

Weber, M. 2004. Philosophy of Experimental Biology. Cambridge University Press.

Weber, M. 2013. "Causal Selection vs Causal Parity in Biology: Relevant Counterfactuals and Biologically Normal Interventions." In Causation in Biology and Philosophy, edited by C. K. Waters, M. Travisano, and J. Woodward. Minneapolis: University of Minnesota Press.

Weber, M. 2017. "Discussion Note: Which Kind of Causal Specificity Matters Biologically?” Philosophy of Science 84 (3): 574-585.

Weber, M. 2018. "Experiment in Biology." The Stanford Encyclopedia of Philosophy (Summer 2018 Edition), edited by Edward N. Zalta. https://plato.stanford.edu/archives/sum2018/entries/biologyexperiment/.

Woodward, J. 2010. "Causation in Biology: Stability, Specificity, and the Choice of Levels of Explanation.” Biology and Philosophy 25: 287-318.

Wu, W., G. Ahlsen, D. Baker, L. Shapiro, and S. L. Zipursky. 2012. "Complementary Chimeric Isoforms Reveal Dscam1 Binding Specificity in Vivo." Neuron 74 (2): 261-268.

Xie, J., N. Wu, A. Brock, G. Spraggon, and P. Schultz. 2004. "The Site-Specific Incorporation of piodo-L-phenylalanine into Proteins for Structure Determination." Nature Biotechnology 22 (10): 1297-1301.

(C) 2019 Author(s)

This is an open-access article distributed under the terms of the Creative Commons Attribution 4.0 International license, which permits anyone to download, copy, distribute, display, or adapt the text without asking for permission, provided that the creator(s) are given full credit.

ISSN 2475-3025 